\title{
GLP-1 secretion is regulated by IL-6 signalling: a randomised, placebo-controlled study
}

\author{
Helga Ellingsgaard ${ }^{1} \cdot$ Eleonora Seelig ${ }^{2,3} \cdot$ Katharina Timper $^{2,4} \cdot$ Michael Coslovsky $^{5} \cdot$ Line Soederlund $^{1}$. \\ Mark P. Lyngbaek ${ }^{1}$. Nicolai J. Wewer Albrechtsen ${ }^{6}$. Arno Schmidt-Trucksäss ${ }^{7}$. Henner Hanssen ${ }^{7}$ - Walter O. Frey ${ }^{8}$. \\ Kristian Karstoft $^{1}$ • Bente K. Pedersen ${ }^{1}$ • Marianne Böni-Schnetzler ${ }^{9}$ - Marc Y. Donath ${ }^{2,9}$
}

Received: 25 July 2019 / Accepted: 1 October 2019 / Published online: 3 December 2019

(C) Springer-Verlag GmbH Germany, part of Springer Nature 2019

\begin{abstract}
Aims/hypothesis IL-6 is a cytokine with various effects on metabolism. In mice, IL-6 improved beta cell function and glucose homeostasis via upregulation of glucagon-like peptide 1 (GLP-1), and IL-6 release from muscle during exercise potentiated this beneficial increase in GLP-1. This study aimed to identify whether exercise-induced IL-6 has a similar effect in humans.

Methods In a multicentre, double-blind clinical trial, we randomly assigned patients with type 2 diabetes or obesity to intravenous tocilizumab (an IL-6 receptor antagonist) $8 \mathrm{mg} / \mathrm{kg}$ every 4 weeks, oral sitagliptin (a dipeptidyl peptidase- 4 inhibitor) $100 \mathrm{mg}$ daily or double placebos (a placebo saline infusion every 4 weeks and a placebo pill once daily) during a 12 week training intervention. The primary endpoints were the difference in change of active GLP-1 response to an acute exercise bout and change in the AUC for the concentration-time curve of active GLP-1 during mixed meal tolerance tests at baseline and after the training intervention.

Results Nineteen patients were allocated to tocilizumab, 17 to sitagliptin and 16 to placebos. During the acute exercise bout active GLP-1 levels were $26 \%$ lower with tocilizumab (multiplicative effect: 0.74 [95\% CI $0.56,0.98$ ], $p=0.034$ ) and 53\% higher with sitagliptin $(1.53[1.15,2.03], p=0.004)$ compared with placebo. After the 12 week training intervention, the active GLP-1 AUC with sitagliptin was about twofold that with placebo $(2.03[1.56,2.62] ; p<0.001)$, while GLP-1 AUC values showed a small non-significant decrease of $13 \%$ at 4 weeks after the last tocilizumab infusion $(0.87[0.67,1.12] ; p=0.261)$. Conclusions/interpretation IL-6 is implicated in the regulation of GLP-1 in humans. IL-6 receptor blockade lowered active GLP1 levels in response to a meal and an acute exercise bout in a reversible manner, without lasting effects beyond IL-6 receptor blockade.

Trial registration Clinicaltrials.gov NCT01073826.
\end{abstract}

Helga Ellingsgaard and Eleonora Seelig are joint first authors.

Electronic supplementary material The online version of this article (https://doi.org/10.1007/s00125-019-05045-y) contains peer-reviewed but unedited supplementary material, which is available to authorised users.

Helga Ellingsgaard

Helga.Ellingsgaard@regionh.dk

1 Centre of Inflammation and Metabolism (CIM)/ Centre for Physical Activity Research (CFAS), Rigshospitalet 7641, Blegdamsvej 9, DK-2100 Copenhagen, Denmark

2 Clinic of Endocrinology, Diabetes and Metabolism University Hospital Basel, Basel, Switzerland

3 University of Cambridge Metabolic Research Laboratories, Wellcome Trust-Medical Research Council Institute of Metabolic Science, Addenbrooke's Hospital, Cambridge, UK
4 Max Planck Institute for Metabolism Research Cologne, Center for Endocrinology, Diabetes and Preventive Medicine (CEDP), University Hospital Cologne, Cologne, Germany

5 Department of Clinical Research, CTU, University of Basel, University Hospital Basel, Basel, Switzerland

6 Department of Clinical Biochemistry, Rigshospitalet University of Copenhagen, Copenhagen, Denmark

7 Sports and Exercise Medicine, Department of Sport, Exercise and Health, University of Basel, Basel, Switzerland

8 Balgrist MoveMed, Swiss Olympic Medical Center, University Hospital Balgrist, Zurich, Switzerland

9 Department Biomedicine, University of Basel, Basel, Switzerland 


\section{Research in context}

\section{What is already known about this subject?}

- Exercise stimulates IL-6 release from skeletal muscle in humans

- Exercise has been found to enhance GLP-1 secretion acutely and following a training intervention in humans

- In mice, exercise-induced GLP-1 secretion is dependent on IL-6 signalling

What is the key question?

- Does a single exercise bout and/or a training intervention lead to an upregulation of active GLP-1 in humans and, if so, is this upregulation dependent on IL-6?

What are the new findings?

- IL-6 receptor blockade (using tocilizumab) lowers meal-stimulated active GLP-1 levels

- During exercise this effect is preserved, but not further increased

- Regulation of active GLP-1 is dependent on IL-6, but not necessarily enhanced by exercise

How might this impact on clinical practice in the foreseeable future?

- These findings may have clinical implications for individuals with diabetes who receive treatment for rheumatoid arthritis with tocilizumab

Funding Danish National Research Foundation. Danish Council for Independent Research. Novo Nordisk Foundation. Danish Centre for Strategic Research in Type 2 Diabetes. European Foundation for the Study of Diabetes. Swiss National Research Foundation.

Keywords Diabetes $\cdot$ Exercise $\cdot$ Glucagon-like peptide- $1 \cdot$ Interleukin- 6 - Obesity

\section{Abbreviations \\ CIM Centre of Inflammation and Metabolism \\ CFAS Centre for Physical Activity Research \\ DPP-4 Dipeptidyl peptidase-4 \\ GIP Glucose-dependent insulinotropic peptide \\ GLP-1 Glucagon-like peptide-1 \\ MMTT Mixed meal tolerance test \\ PYY Peptide YY}

\section{Introduction}

Exercise has the potential to improve glucose homeostasis and influence nearly all stages of type 2 diabetes. It can delay and prevent the onset of the disease [1], improve glycaemic control [2] and replace glucose-lowering medication [3]. How exercise improves glucose homeostasis in humans is not fully understood; in particular, beta cell adaptations to exercise remain to be clarified.

IL-6 is a pleiotropic cytokine that affects metabolism [4]. Moreover, IL-6 is a myokine produced by skeletal muscle and is released into the circulation in response to exercise [5, 6]. Systemic concentrations of IL-6 increase acutely following an exercise bout [6]. Levels of the incretin hormone glucagon-like peptide-1 (GLP-1) have also been reported to increase acutely in response to an exercise bout [7]. Classically, however, GLP-1 secretion is stimulated by nutrients when these reach the intestinal L cells [8]; biologically active GLP-1 subsequently acts on pancreatic beta cells to potentiate insulin secretion [9] and on pancreatic alpha cells to inhibit glucagon secretion [10]. It has been suggested that exercise training can improve the secretory capacity of L cells $[11,12]$.

Our own rodent studies have highlighted a skeletal muscle/ intestinal crosstalk and revealed that exercise-induced GLP-1 secretion requires IL-6 [13]. Furthermore, both in mice and humans, an acute increase in systemic IL-6 (following administration of IL-6) improved glucose tolerance, and IL-6 improved glucose tolerance through GLP-1-dependent stimulation of insulin secretion in mice [13]. In humans, IL-6 improved postprandial glycaemia via inhibition of gastric emptying [14]. Whether endogenous IL-6 is involved in skeletal muscle/ 
intestinal crosstalk in humans has yet to be determined. We hypothesised that exercise-induced GLP-1 is dependent on IL-6 and, moreover, that exercise-induced GLP-1 may have lasting effects on beta cell function following a training intervention. Furthermore, we hypothesised that combining exercise with a dipeptidyl peptidase-4 (DPP-4) inhibitor will prolong the halflife of exercise-induced GLP-1, thereby potentiating the effect of GLP-1, leading to beta cell adaptations superior to those achieved by exercise alone. We therefore tested whether a single bout of exercise and a training intervention enhances GLP-1 secretion, and whether this upregulation is dependent on IL-6. Obese individuals and individuals with type 2 diabetes performed an acute exercise bout followed by a 12 week physical training intervention, during which time they received an IL-6 receptor antagonist (tocilizumab), a DPP-4 inhibitor (sitagliptin) or placebo.

\section{Methods}

\section{Study design and participants}

This randomised, placebo-controlled, double-blind, multicentre study included 54 individuals with either type 2 diabetes mellitus or obesity. From February 2010 to November 2015 participants were enrolled at the University Hospitals of Basel and Zurich, Switzerland, and at the Centre of Inflammation and Metabolism (CIM), Centre for Physical Activity Research (CFAS), Rigshospitalet, University of Copenhagen, Denmark. The main study personnel at all study centres were trained at University Hospital Zurich to ensure similar performance across all three trial centres.

Participants were eligible if they were aged 18-75 years, were obese (BMI $\geq 30$ and $\left.\leq 40 \mathrm{~kg} / \mathrm{m}^{2}\right)$ and non-diabetic or had a diagnosis of type 2 diabetes according to ADA criteria for at least 3 months. Key exclusion criteria were glucose-lowering drugs other than metformin, diabetes duration $\geq 5$ years, any inflammatory, infectious or immunosuppressive disease, treatment with corticosteroids, nonsteroidal anti-inflammatory drug or aspirin, pregnancy or breastfeeding. All participants provided written informed consent.

The study was approved by the Swiss ethical committees of the participating hospitals, Swissmedic and the regional ethics committee, Copenhagen, Denmark (H-4-2013-098), and was conducted in accordance with the guidelines for Good Clinical Practice and the Declaration of Helsinki. The results have been reported in accordance with the CONSORT guidelines and the trial is registered at Clinicaltrials.gov NCT01073826.

\section{Randomisation and treatment}

Participants were randomised in a simple 1:1:1 ratio, stratified according to whether they were diabetic or obese, to receive either tocilizumab (RoActemra, Roche Pharma), an IL-6 receptor antibody, $8 \mathrm{mg} / \mathrm{kg}$ body weight administered i.v. every 4 weeks, or sitagliptin (Januvia, MSD Merck Sharp \& Dohme), a DPP-4 inhibitor, $100 \mathrm{mg}$ taken orally once daily, or placebos (a placebo infusion every 4 weeks and a placebo pill once daily). To mask treatment allocation, participants in the sitagliptin group were given a placebo infusion every 4 weeks, those receiving tocilizumab had a placebo pill once daily, and the placebo group received both placebos. An equal number of participants with type 2 diabetes and obese participants were randomised into each of the three study arms. Randomisation was performed by an external statistician. Participants as well as study personnel were blinded to the medication allocation. Participants performed an initial exercise bout $(60 \mathrm{~min})$ followed by a 12 week training intervention.

\section{Study assessment}

All participants had a screening visit, followed by five study visits. The study design is depicted in Electronic supplementary material (ESM) Fig. 1. At baseline a standardised mixed meal tolerance test (MMTT) was performed after an overnight fast. Baseline blood samples were taken before and during a $2 \mathrm{~h}$ MMTT with $360 \mathrm{ml}$ of Boost containing $62 \mathrm{~g}$ carbohydrates, $15 \mathrm{~g}$ protein and $6 \mathrm{~g}$ fat (Nestlé, Switzerland). Afterwards, a bicycle ergometer test was performed to determine maximum oxygen consumption $\left(\dot{V} \mathrm{O}_{2 \max }\right)$. One week later, participants returned to the research facilities after an overnight fast. Fasting blood samples were taken before the study drugs were given. At the end of a $1 \mathrm{~h}$ i.v. infusion of the study drug, blood sampling was repeated before participants ingested a mixed meal ( $360 \mathrm{ml}$ of Boost). One hour after meal ingestion participants performed an acute exercise bout for 60 min on a bicycle ergometer at $75 \%$ of $\dot{V} \mathrm{O}_{2 \max }$. Further blood samples were taken every 10 min during the exercise bout. Blood sampling was continued for another $30 \mathrm{~min}$ after completion of the exercise bout. Participants were then given instructions for unsupervised bicycle training consisting of a 5 min warm up followed by $45 \mathrm{~min}$ at $75 \% \dot{\mathrm{V}} \mathrm{O}_{2 \max }$ three to five times a week. Heart rate monitors (Polar Watch, Polar, UK) and training diaries were dispensed, and participants were asked to take one tablet of study drug once a day. After 4 and 8 weeks, participants returned to the research facilities for infusion of the study drug, as well as for safety and compliance assessments. After completing the 12 week training intervention, the same assessments as performed at the baseline visit were repeated.

All blood samples analysed for gastrointestinal hormones were treated identically at all three trial centres and were centrally measured by Roche Diagnostics. Active GLP-1 [(7-36) $\left.\mathrm{NH}_{2}\right]$, inactive and total glucose-dependent insulinotropic peptide (GIP) and peptide YY [PYY; PYY (1-36)] were measured using the 


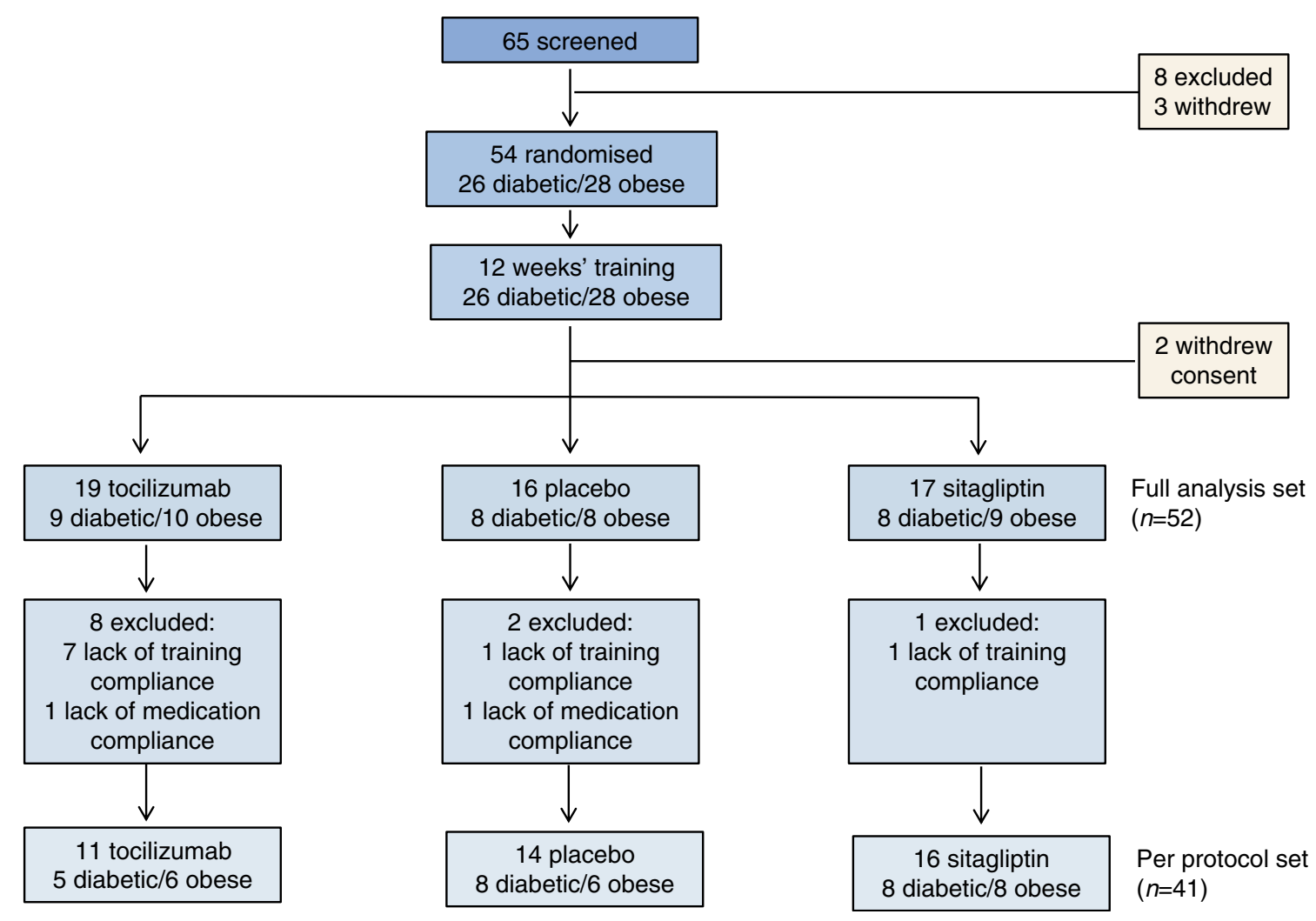

Fig. 1 Flow chart of enrolment, randomisation and treatment

IMPACT chip (Roche Diagnostics, Bernried am Starnberger See, Germany). Glucagon, insulin, proinsulin, and C-peptide were assessed using ELISA assays (Mercodia, Sweden). IL-6, IL-8, $\mathrm{TNF}-\alpha$ were measured using commercially available assays (Meso Scale Diagnostics, USA), IL-1 receptor antagonist (IL1Ra) using an ELISA assay (R\&D Systems, Minneapolis, MN, USA). Apart from the gastrointestinal peptides, all the abovementioned parameters including high-sensitivity C-reactive protein (hs-CRP) and cortisol were centrally measured at the routine laboratory, Department of Clinical Biochemistry, Rigshospitalet Copenhagen University Hospital. Glucose, $\mathrm{HbA}_{1 \mathrm{c}}$, lipids and safety blood parameters (blood counts, liver and kidney function tests) were analysed in the routine central laboratory units of the University Hospitals in Basel and Zurich, Switzerland, and at the department of Clinical Biochemistry, Rigshospitalet, Denmark. The reported HOMA index was calculated according to the method of Matthews et al [15], while insulin secretion index was derived from the method of Phillips et al [16]. DPP-4 activity was assessed using a previously described assay [17] and DPP-4 protein concentrations using a commercially available assay (catalogue no. DC260B, R\&D Systems).

\section{Study endpoints}

The primary outcomes were the change of active GLP-1 in response to an acute exercise bout and the change in the AUC for the concentration-time curve of active GLP-1 during an MMTT at baseline and after a 12 week training intervention. Predefined secondary endpoints were beta cell function, glucose homeostasis, total and inactive GIP, PYY, glucagon, insulin, cortisol, IL-6, IL-8, IL-1Ra, TNF- $\alpha$ and hs-CRP during an acute exercise bout as well as the change from baseline to 12 weeks' training. Additional secondary endpoints were change in creatine kinase, fasting lipid profile and liver values between baseline and after 12 weeks of training.

\section{Statistical analysis}

The full analysis set included all participants to whom a treatment group was randomly assigned. The per protocol set included all individuals of the full analysis population set who had both baseline and 12 week GLP-1 AUC values and had no critical protocol violations. Critical protocol violations were defined as failure to attend for infusion of study drug or placebo, failure to attend the MMTT at the end of the 12 weeks, fewer than 26 training sessions or failure to take oral study drug (sitagliptin) on more than 12 days.

The analysis of active GLP-1 levels during the acute exercise bout was performed using a linear mixed-effects model. Participant ID and a random slope were included as random effects to account for intra-patient correlation. As fixed predictors, the model included time, treatment group and the 
interaction between time and treatment group. The interaction was removed from the model if it was not significant $(p<0.05)$ to improve interpretability of main effects. To test for differences in active GLP-1 levels over time between participants with type 2 diabetes and obese participants, the model was fitted once again, this time including an interaction term between time and participant type. Active GLP-1 levels in response to the study drug before the exercise bout were compared using an ANCOVA model with baseline levels as covariate. Active GLP-1 levels in response to a meal before the exercise bout and during a resting period after the exercise were analysed using linear models with treatment group and time, and interaction between treatment group and time as predictors.

The change in GLP-1 AUC after a 12 week training intervention was compared between study arms using an analysis of covariance approach with baseline GLP-1 AUC as covariate. AUCs were calculated based on the trapezoid rule. For all analysis the natural logarithm of AUC values was taken. The main analysis was performed using the full analysis set, with multiple imputations based on chained equations used to account for missing AUC values. To examine the sensitivity of the results to the missing values mechanism the analysis was repeated on the per protocol set. The appropriateness of the imputed data was examined before analysis. Differences in treatment effect between participant types, i.e. diabetic and obese participants, were examined by adding an interaction term between participant type and treatment group to the primary model. A subgroup analysis was done in the per protocol set, where the primary model (ANCOVA) was repeated once for diabetic and once for obese participants only. For all secondary endpoints, summary statistics are reported. Results are expressed as estimates of the group differences in change from baseline with a 95\% CI. For descriptive statistics we summarise categorical variables as counts (percentages) and continuous variables as mean \pm SD. For the two main outcomes, multiple comparisons between study arms are performed and adjusted $p$ values reported using Holm's correction [18].

For the main analysis a $p$ value of $<0.05$ was considered significant. Data were analysed using $\mathrm{R}$ version 3.3.3 (R Software Foundation, Vienna, Austria) for mixed effect models; the nlme package (version nlme 3.1.131) was used [19].

\section{Results}

Between February 2010 and November 2015, 54 participants were enrolled in the study. Seven patients were enrolled at the University Hospital Zurich, 29 at the University Hospital Basel and 18 at the Rigshospitalet, Denmark. Of these 54, two participants (one obese, one with diabetes) withdrew their consent after randomisation. Of the remaining 52 participants, 27 participants were obese but did not have diabetes and 25 participants had a diagnosis of type 2 diabetes. The 52 participants were allocated to tocilizumab $(n=19)$, sitagliptin $(n=$ $17)$ or placebo $(n=16)$ and were included in the full analysis set (Fig. 1). A total of 41 participants completed the study without critical protocol violations and were included in the per protocol set. Of note, seven participants in the tocilizumab group were excluded before unblinding due to lack of compliance to training, whereas in the placebo and sitagliptin groups one participant per group was excluded due to lack of training compliance. Baseline characteristics were similar between treatment groups (Table 1). Baseline characteristics according to participant group (obese or diabetic) are presented in ESM Table 1 . $\mathrm{HbA}_{1 \mathrm{c}}$ levels were $35.83 \pm 3.65 \mathrm{mmol} / \mathrm{mol}(5.43 \pm$ $0.33 \%)$ in obese participants and $49.48 \pm 9.39 \mathrm{mmol} / \mathrm{mol}$ $(6.68 \pm 0.68 \%)$ in patients with type 2 diabetes. There were 14 participants on metformin (dose: $1.83 \pm 0.5 \mathrm{mg}$ ) and these were evenly randomised to the three study arms (placebo 5, tocilizumab 5, sitagliptin 4).

\section{Exercise bout}

During exercise bout The development of active GLP-1 over exercise time did not depend on treatment arm, since the interaction between time and treatment group was not significant $(p=0.315)$; this interaction was therefore subsequently removed from the model. Active GLP-1 values during the exercise bout were $26 \%$ lower in the tocilizumab group (multiplicative effect: 0.74 [95\% CI 0.56, 0.98], $p=0.034$ ) and $53 \%$ higher in the sitagliptin group $(1.53[1.15,2.03]$, $p=0.004$ ) compared with the placebo group (Fig. 2a, ESM Table 2). In addition, the effect of time was small, with a multiplicative effect of $10 \mathrm{~min}$ being almost 1 (0.99 [0.97, $1.01], p=0.303$ ). However, the development of active GLP1 during exercise did differ between obese patients and those with type 2 diabetes ( $p$-interaction $<0.001$ ), but there was no strong change in the effects of treatment groups (Fig. 2b, c). Whereas active GLP-1 levels slightly reduced over time $(0.95$ $[0.93,0.98], p<0.001)$ in participants with type 2 diabetes, the effect in obese participants was a slight increase in GLP-1 levels, indicated by the interaction $(1.08$ [1.04, 1.12], $p<0.001)$.

Before and after the acute exercise period In the time period between receiving the study drug $(-120 \mathrm{~min})$ and having the meal ( $-60 \mathrm{~min})$ the change in active GLP-1 levels differed between treatment groups, as indicated by the significant interaction term $(p<0.001)$. Active GLP-1 levels decreased in a similar way in the placebo group (multiplicative effect: $0.79[95 \%$ CI $0.69,0.90])$ and the tocilizumab group $(0.80$ $[0.72,0.90]$; interaction test $p=0.831)$. In contrast, in the sitagliptin group the change in active GLP-1 level differed 
Table 1 Baseline characteristics. Data based on available data from the full analysis set $(n=52)$

\begin{tabular}{|c|c|c|c|}
\hline Characteristic & Placebo $(n=16)$ & Sitagliptin $(n=17)$ & Tocilizumab $(n=19)$ \\
\hline Age (years) & $51.6( \pm 14.96)$ & $50.0( \pm 16.6)$ & $44.3( \pm 15.88)$ \\
\hline Female sex $(\%)$ & 50.0 & 64.7 & 47.4 \\
\hline BMI $\left(\mathrm{kg} / \mathrm{m}^{2}\right)$ & $29.6( \pm 4.3)$ & $31.6( \pm 6.3)$ & $33.5( \pm 5.2)$ \\
\hline Systolic BP (mmHg) & $131.6( \pm 16.6)$ & $135.1( \pm 15.8)$ & $131.2( \pm 16.5)$ \\
\hline Diastolic BP (mmHg) & $79.7( \pm 10.2)$ & $83.8( \pm 12.5)$ & $82.4( \pm 12.8)$ \\
\hline$\dot{V} O_{2 \max }\left(\mathrm{ml} \mathrm{min} \min ^{-1} \mathrm{~kg}^{-1}\right)$ & $25.1( \pm 6.7)$ & $24.6( \pm 6.5)$ & $25.2( \pm 6.6)$ \\
\hline $\mathrm{HbA}_{1 \mathrm{c}}(\mathrm{mmol} / \mathrm{mol})$ & $44.36( \pm 12.0)$ & $41.08( \pm 7.9)$ & $41.85( \pm 9.5)$ \\
\hline $\mathrm{HbA}_{1 \mathrm{c}}(\%)$ & $6.21( \pm 1.10)$ & $5.91( \pm 0.72)$ & $5.98( \pm 0.87)$ \\
\hline Total cholesterol $(\mathrm{mmol} / \mathrm{l})$ & $4.63( \pm 0.94)$ & $4.64( \pm 0.99)$ & $4.51( \pm 0.64)$ \\
\hline LDL-cholesterol (mmol/l) & $2.75( \pm 0.84)$ & $2.88( \pm 0.9)$ & $2.60( \pm 0.62)$ \\
\hline HDL-cholesterol (mmol/l) & $1.34( \pm 0.41)$ & $1.24( \pm 0.21)$ & $1.05( \pm 0.24)$ \\
\hline Triacylglycerol (mmol/l) & $1.34( \pm 0.55)$ & $1.24( \pm 0.75)$ & $1.73( \pm 0.98)$ \\
\hline hs-CRP (nmol/l) & $20.6( \pm 13.6)$ & $26.8( \pm 25.3)$ & $31.8( \pm 28.5)$ \\
\hline $\mathrm{HbA}_{1 \mathrm{c}}(\mathrm{mmol} / \mathrm{mol})$ in type 2 diabetes & $53.65( \pm 11.6)$ & $45.88( \pm 7.5)$ & $49.39( \pm 8.6)$ \\
\hline $\mathrm{HbA}_{1 \mathrm{c}}(\%)$ in type 2 diabetes & $7.06( \pm 1.06)$ & $6.35( \pm 0.69)$ & $6.67( \pm 0.79)$ \\
\hline $\mathrm{HbA}_{1 \mathrm{c}}(\mathrm{mmol} / \mathrm{mol})$ in obesity & $36.27( \pm 3.5)$ & $35.06( \pm 3.2)$ & $36.16( \pm 4.6)$ \\
\hline $\mathrm{HbA}_{1 \mathrm{c}}(\%)$ in obesity & $5.47( \pm 0.32)$ & $5.36( \pm 0.29)$ & $5.46( \pm 0.42)$ \\
\hline
\end{tabular}

significantly (interaction test $p<0.001$ ) from that in the placebo group, with an overall increase in active GLP-1 levels (multiplicative effect: $1.42[1.25,1.61]$ ) (Fig. 2a). In response to the meal ( -60 to $0 \mathrm{~min}$ ) the effect of treatment with sitagliptin was a further increase in active GLP-1 (treatment effect $\times$ time effect: $2.25 \times 1.51=3.398$ ), while tocilizumab was shown to further inhibit the increase in active GLP-1 $(0.82 \times 2.25=1.845)$ compared with placebo. There was no evidence that the change in active GLP-1 was dependent on time (interaction test $p=0.9$ ). Interestingly, participants with type 2 diabetes receiving placebo showed a blunted increase in active GLP-1 levels in response to the meal compared with their obese counterparts (Fig. 2b, c); these findings were not tested for statistical significance. During the resting period after the exercise bout (60-90 $\mathrm{min}$ ), active GLP-1 levels initially increased in all treatment groups (time point at 75 min: 1.16 [1.03, 1.31], $p=0.014$ ) before returning to levels similar to those measured at the end of the exercise (time point at 90 min: 0.93 [0.83, 1.05], $p=0.244$ ); data are multiplicative effects with respect to baseline for all patients (interaction test $p=0.453$ ). Participants treated with sitagliptin showed $43 \%$ higher levels of active GLP-1 compared with those who received placebo $(1.43[1.10,1.87], p=0.01)$, whereas participants treated with tocilizumab showed a $23 \%$ reduction in active GLP-1 levels compared with those in the placebo group $(0.77[0.60,1.01], p=0.061)$, but this difference did not reach significance. Descriptive summary statistics and figures for all the following secondary endpoints measured during the exercise bout are shown in ESM Figs 2 and 3. During the exercise bout, PYY behaved like active GLP-1, i.e. it was higher with sitagliptin and lower with tocilizumab, but this was not tested for statistical significance. Interestingly, this effect was again observed in the obese participants rather than in the participants with type 2 diabetes (ESM Fig. 2). Whereas inactive GIP appeared to rise during the meal and exercise bout in the placebo and tocilizumab groups, inactive GIP remained low in the sitagliptin group (ESM Fig. 2).

No obvious differences could be seen in the shape of the curves of glucose, insulin and C-peptide between treatment groups (Fig. 3a-f). Neither did the distribution of the curves of glucagon and cortisol suggest any distinct difference between treatments (Fig. $3 \mathrm{~g}-\mathrm{j}$ ). On visual inspection of the data from the two participant subgroups, participants with type 2 diabetes responded to sitagliptin with an increase in insulin and C-peptide as well as glucagon, while cortisol levels were lower than those with type 2 diabetes who received tocilizumab or placebo; these findings were not tested for statistical significance. IL-6 levels were overall higher with tocilizumab and increased in response to exercise in all groups (ESM Fig. 4a, b). There was no apparent difference in IL-8, TNF- $\alpha$, IL-1Ra between the three treatment groups (ESM Fig. 4c-e). Protein levels of DPP-4 and DPP-4 activity were assessed in plasma samples from $n=3$ obese participants. The plasma concentration of DPP-4 protein was unaffected by time or treatment. DPP-4 activity also remained unchanged in the placebo and tocilizumab groups and was, as expected, reduced in the sitagliptin group (ESM Fig. 5).

\section{Training intervention}

In the full analysis set, the increase in active GLP-1 AUC from baseline to 12 weeks in the sitagliptin group was about 

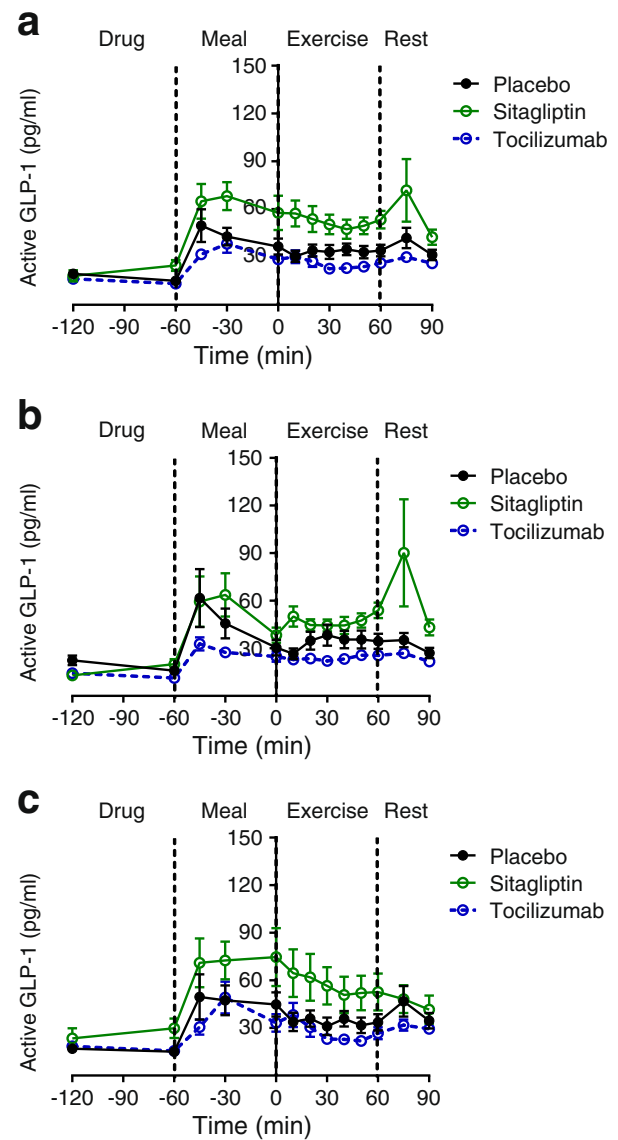

Fig. 2 DPP-4 inhibition (with sitagliptin) and IL-6 receptor antagonism (with tocilizumab) differentially regulate active GLP-1 during an acute exercise bout. (a) Active GLP-1 values during the exercise bout are $26 \%$ lower with tocilizumab (multiplicative effect: 0.74 [95\% CI 0.56, 0.98], $p=0.034)$ and $53 \%$ higher with sitagliptin $(1.53$ [1.15, 2.03], $p=0.004)$ compared with placebo (obese participants and participants with type 2 diabetes combined). (b) In obese participants active GLP-1 levels slightly increase $(1.08$ [1.04, 1.12], $p<0.001)$ during exercise, compared with baseline. (c) In participants with type 2 diabetes, active GLP-1 decreased over time $(0.95$ [0.93, 0.98], $p<0.001)$ compared with baseline. Data represent mean values, error bars indicate SEM based on the full analysis set

twofold that in the placebo group (multiplicative effect: 2.03 [95\% CI 1.56, 2.62]; $p<0.001$ ) (ESM Table 3). In contrast, active GLP-1 AUC values showed a small non-significant decrease of $13 \%$ in the tocilizumab group compared with the placebo group $(0.87[0.67,1.12] ; p=0.261)$. The effect of treatment was not influenced by baseline active GLP-1 AUC values (interaction test $p=0.285$ ). Analysis in the per protocol set produced similar results (ESM Table 4).

The effects of treatment with sitagliptin and tocilizumab were similar for participants with type 2 diabetes and obese participants (interaction test $p=0.777$ ). In both participants with type 2 diabetes and in obese participants, the increase in log-transformed active GLP-1 AUC at 12 weeks in the sitagliptin group was about twofold that in the placebo group (obese group: 2.11 [1.34, 3.34], $p=0.003$; type 2 diabetic group: 2.03 [1.51, 2.72]; $p<0.001)$. There was no significant change in response to tocilizumab treatment in both subgroups (obese group: $0.84[0.51,1.36], p=0.452$; type 2 diabetic group: $1.12[0.80,1.56] ; p=0.493)$. Subgroup analysis was performed in the per protocol set (ESM Table 5). Timeconcentration curves for active GLP-1 in response to the mixed meal are shown in Fig. 4. There was no evidence that male or female sex affected GLP-1 secretion (data not shown).

Descriptive summary statistics and figures are shown in ESM Figs 6 and 7. As in the exercise bout, inactive GIP seemed lower with sitagliptin treatment after 12 weeks of training, while remaining stable in the placebo and tocilizumab group. On visual inspection, total GIP and PYY were similar between groups. PYY levels appeared to be lower in the obese participants; however, these findings were not tested for statistical significance.

No obvious differences between groups were observed in glucose homeostasis when comparing glucose (Fig. 5a, b), insulin (Fig. 5c, d), C-peptide (Fig. 5e, f), and glucagon (Fig. 5g, h). All groups lost weight (ESM Table 6), but changes in body weight were not tested for statistical significance.

$\mathrm{HbA}_{1 \mathrm{c}}$ was lower in obese participants compared with individuals with type 2 diabetes, but there was no obvious difference between treatment groups. Moreover, both insulinogenic index and HOMA-IR remained unchanged in the obese group (Fig. 5i,k, ESM Table 7) as well as in patients with type 2 diabetes (Fig. 5 j,1, ESM Table 7).

On visual inspection IL-6 increased with tocilizumab, while other cytokines (IL-8, TNF- $\alpha$, IL-1Ra) apparently did not change between groups (ESM. Fig. 7); however, this was not tested for statistical significance.

Descriptive summary statistics of hs-CRP, creatine kinase, lipid profile and liver values before and after the 12 week training period did not indicate any differences on visual inspection (data not shown).

$\dot{V} O_{2 \max }$ increased from $25.1( \pm 6.7)$ to $26.5( \pm 7.2)$ $\mathrm{ml} \mathrm{kg}{ }^{-1} \mathrm{~min}^{-1}$ in the placebo group, from $24.6( \pm 6.5)$ to $27.0( \pm 6.6) \mathrm{ml} \mathrm{kg}^{-1} \mathrm{~min}^{-1}$ with sitagliptin and from 25.2 $( \pm 6.6)$ to $28.2( \pm 7.9) \mathrm{ml} \mathrm{kg}^{-1} \mathrm{~min}^{-1}$ with tocilizumab, respectively.

\section{Adverse events}

Overall 116 adverse events occurred (ESM Table 8). In addition, in the tocilizumab group one participant developed a seronegative rheumatoid arthritis, which was considered a serious adverse event but unlikely to be due to study medication. An adverse event was experienced by $70.6 \%$ of participants in the placebo group, $64.7 \%$ in the sitagliptin group and $77.8 \%$ in the tocilizumab group. There was no significant association between groups and presence of adverse events $(p \sim 1)$. 
Fig. 3 Effects of DPP-4 inhibition (with sitagliptin) and IL-6 receptor antagonism (with tocilizumab) on blood glucose and pancreatic hormones during an acute exercise bout. No differences between treatment groups were seen for $(\mathbf{a}, \mathbf{b})$ blood glucose, $(\mathbf{c}, \mathbf{d})$ insulin, $(\mathbf{e}, \mathbf{f}) \mathrm{C}$ peptide, $(\mathbf{g}, \mathbf{h})$ glucagon and $(\mathbf{i}, \mathbf{j})$ cortisol in obese participants (a, c, $\mathbf{e}, \mathbf{g}, \mathbf{i})$ and participants with type 2 diabetes $(\mathbf{b}, \mathbf{d}, \mathbf{f}, \mathbf{h}, \mathbf{j})$. Data represent mean values, error bars indicate SEM based on the full analysis set
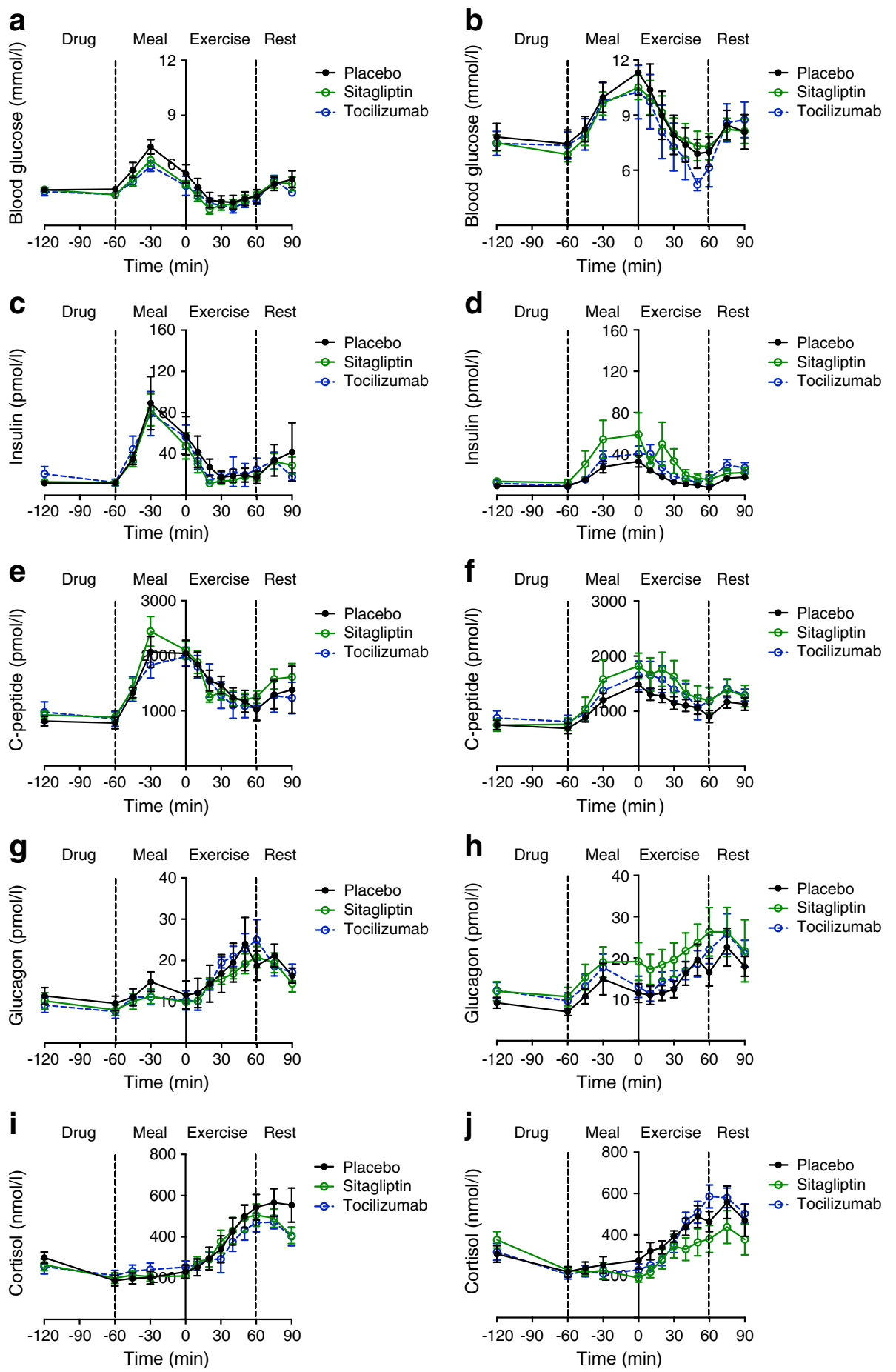

\section{Discussion}

In the present study we show that IL-6 is implicated in the regulation of GLP-1. Tocilizumab, an IL-6 receptor antagonist, used to treat patients with rheumatoid arthritis, lowered active GLP-1 concentrations in response to a meal and an acute exercise bout. In contrast to tocilizumab, sitagliptin led to a rise in active GLP-1 levels in response to a meal, while no further increase was observed during exercise. These findings indicate that in this acute setting, levels of active GLP-1 are partly dependent on IL-6 but are not necessarily enhanced by exercise.

Upon secretion, active GLP-1 is rapidly inactivated by DPP-4 [20]. In addition to changes in activity, the amount of DPP-4 is also variable [21]. Tocilizumab did not affect DPP-4 activity or the amount of DPP-4 protein in plasma, so the 

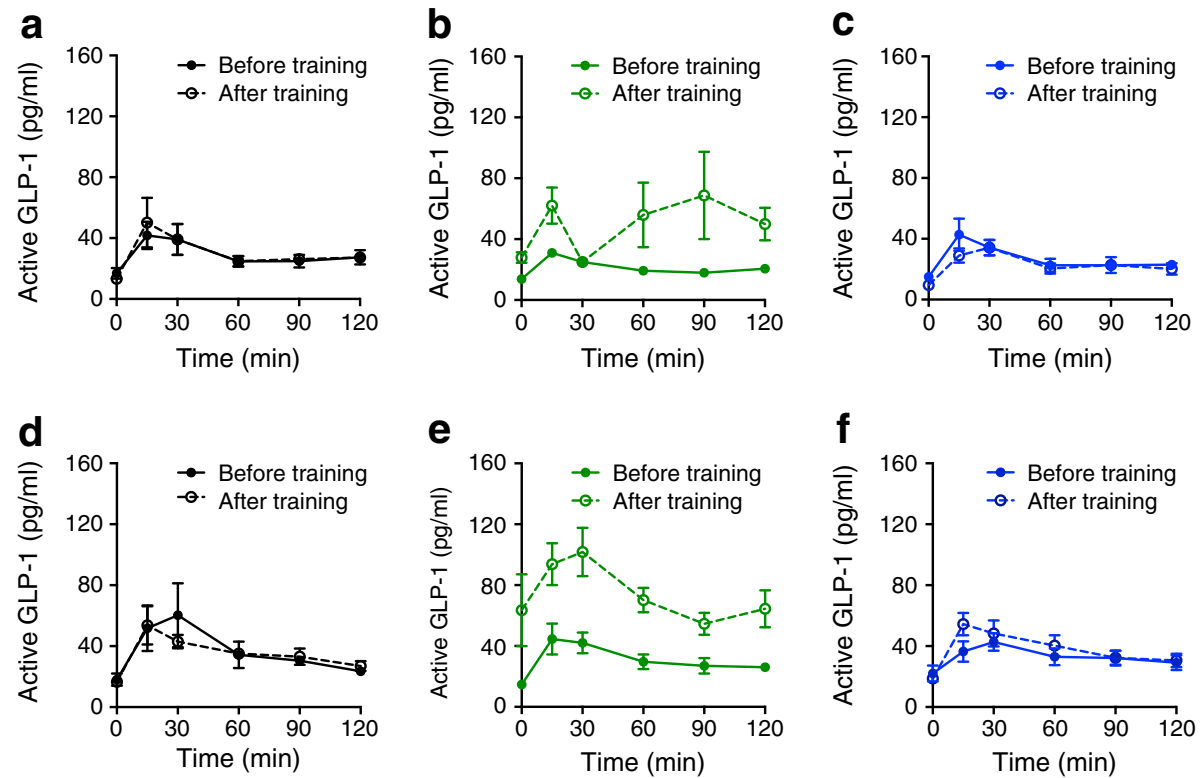

Fig. 4 Effect of training in combination with DPP-4 inhibition (with sitagliptin) or IL-6 receptor antagonism (with tocilizumab) on active GLP-1. Measurements during an MMTT before and after a 12 week training intervention (time point 0 represents the time right before the meal). The increase in active GLP-1 AUC from baseline to 12 weeks is higher with sitagliptin compared with placebo $(p<0.001)$, and lower with tocilizumab compared with placebo (although this was not significant; $p=0.261$ ) (see ESM Table 3). AUC values were calculated from

question remains as to whether the effect of tocilizumab on active GLP-1 is an effect on secretion or interaction with other proteases involved in the degradation of GLP-1 [22].

Subgroup analysis of the acute exercise bout showed that the treatment effects in obese participants and patients with type 2 diabetes were comparable, i.e. tocilizumab lowered and sitagliptin enhanced active GLP-1, but there was an overall discrepancy between the diabetes and the obese groups regarding the regulation of active GLP-1. In participants with type 2 diabetes (on placebo) the increase in active GLP-1 levels in response to the meal was blunted; active GLP-1 levels decreased in diabetic participants during the exercise bout but increased in the obese individuals. A difference in GLP-1 response between individuals with type 2 diabetes and obese individuals has previously been described [23]. Whether this discrepancy is a consequence of progressive L cell failure is not fully understood. Exerciseinduced IL-6 increased to a similar level in both subgroups and does therefore not underlie the discrepancy in GLP-1 secretion.

It is noteworthy that PYY, another $\mathrm{L}$ cell product and one that acts as a substrate for DPP-4 [24], was regulated similarly to active GLP-1. Interestingly, the treatment effect on PYY seemed more pronounced in obese individuals than in participants with type 2 diabetes. These findings could indicate a signalling impairment of $\mathrm{L}$ cells in individuals with type 2 diabetes. Furthermore, IL-6 blockade did not seem to influence the K cell-derived incretin GIP. This supports an L cell-specific effect of IL-6 that is not limited to active GLP-1. measurments during the MMTT. For obese participants (a-c) and participants with type 2 diabetes $(\mathbf{d}-\mathbf{f})$ the treatment effect of sitagliptin and tocilizumab on meal-induced levels of active GLP-1 is similar (interaction test, $p=0.777$ ) (see also ESM Table 5). Black data points (a, d), placebo; green data points (b, e), sitagliptin; blue data points $(\mathbf{c}, \mathbf{f})$, tocilizumab. Data represent mean values, error bars indicate SEM based on the full analysis set

We recently reported that IL-6 is implicated in the regulation of gastric emptying rate [14]. IL-6 receptor blockade acutely increased gastric emptying during a post-exercise meal in healthy men. This increased gastric emptying was associated with increased secretion of GLP-1 (total) and GIP (total), probably a direct consequence of the accelerated gastric emptying [25]. We did not assess gastric emptying and therefore do not know whether IL-6 receptor blockade also regulates gastric emptying in the absence of an exercise bout.

In addition to the acute effect of IL-6 on GLP-1 we investigated the impact of a 12 week treatment period during a training intervention. While increased meal-stimulated GLP1 secretion following an exercise training intervention has previously been reported [12], in the present study this was observed only when combining exercise training and sitagliptin. Although this effect seems mainly driven by sitagliptin, given the pharmacokinetics of this DPP-4 inhibitor $[26,27]$, we cannot exclude the possibility that a mild exercise-driven effect is emphasised in the presence of sitagliptin.

Overall, IL-6 receptor antagonism had no clinically relevant effects on glycaemia. Keeping in mind the multiple metabolic effects of IL-6 that may increase or decrease glycaemia, it is conceivable that the resulting effect is neutral. However, in some individuals with more pronounced defects in insulin secretion or sensitivity, one or the other effect of IL-6 may have a dominating effect. Supporting this hypothesis, 
a

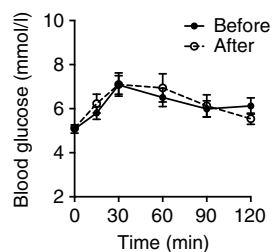

C
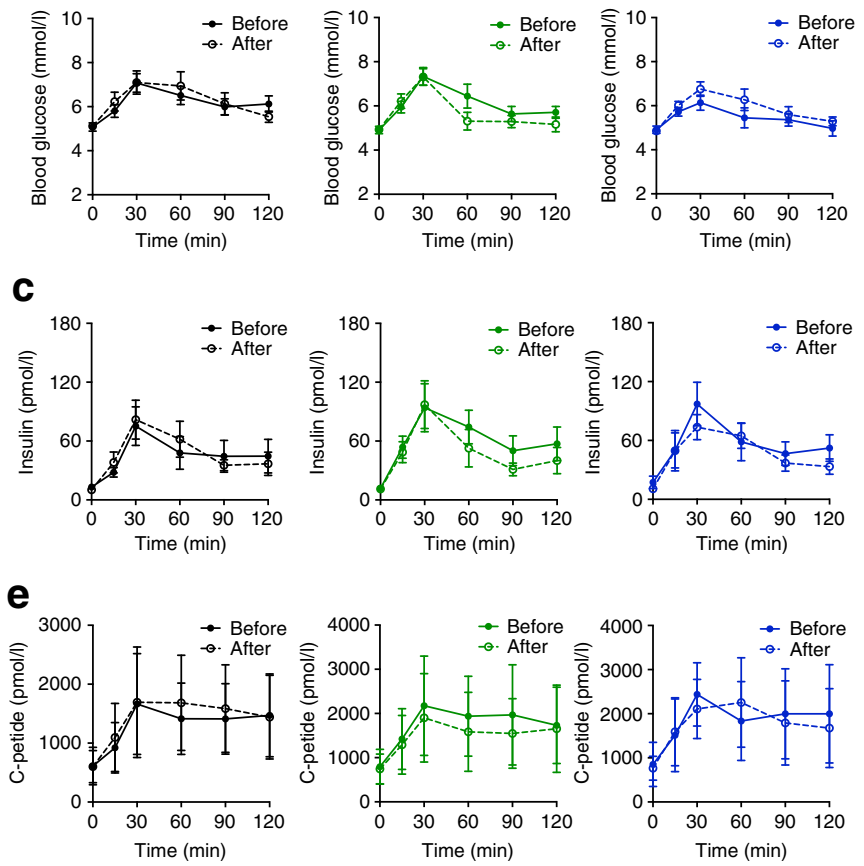

g
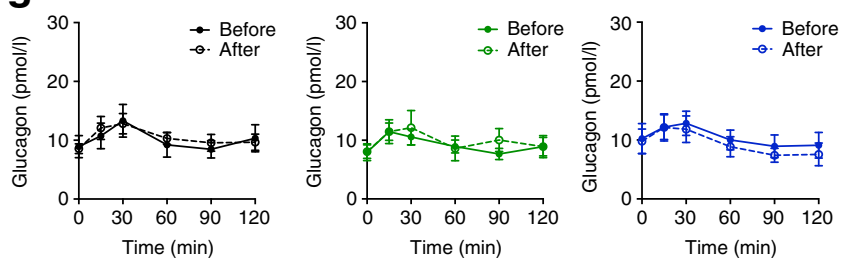

i
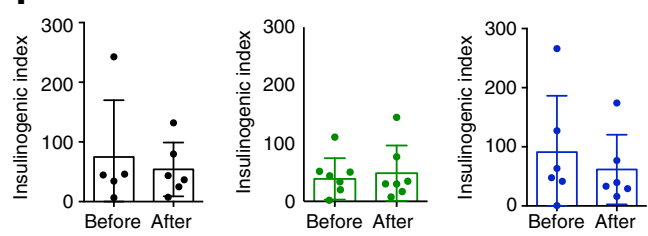

j
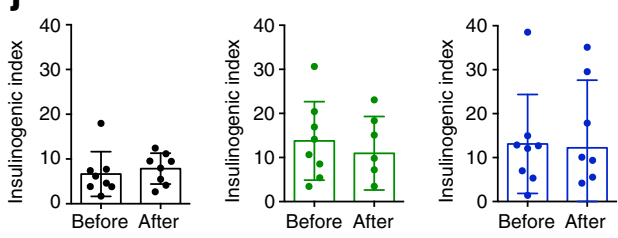

k
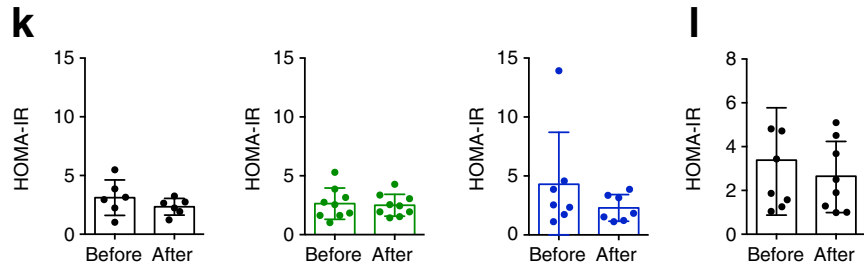

Fig. 5 Effect of training in combination with DPP-4 inhibition (with sitagliptin) or IL-6 receptor antagonism (with tocilizumab) on glycaemic control. (a-h) Measurements during an MMTT before and after a 12 week training intervention in the whole population (full analysis set) are similar in participants with obesity (a, $, \mathbf{c}, \mathbf{e}, \mathbf{g})$ and in those with type 2 diabetes (b, $\mathbf{d}, \mathbf{f}, \mathbf{h})$ for blood glucose $(\mathbf{a}, \mathbf{b})$, insulin $(\mathbf{c}, \mathbf{d})$, C-peptide $(\mathbf{e}, \mathbf{f})$ and

tocilizumab numerically slightly impaired glucose homeostasis in obese individuals, while it improved it in individuals with type 2 diabetes, although these findings were not tested for statistical significance.

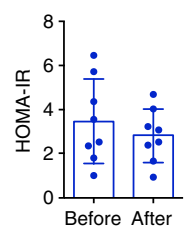
data points, tocilizumab b
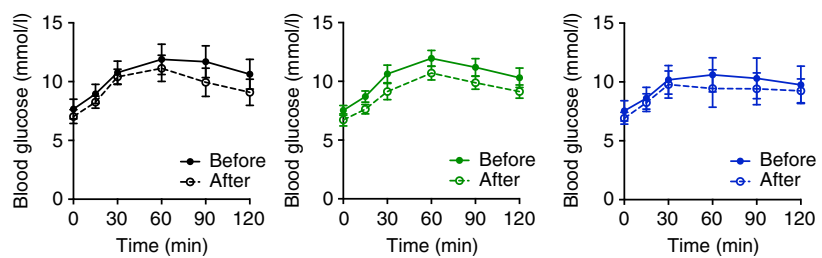

\section{d}
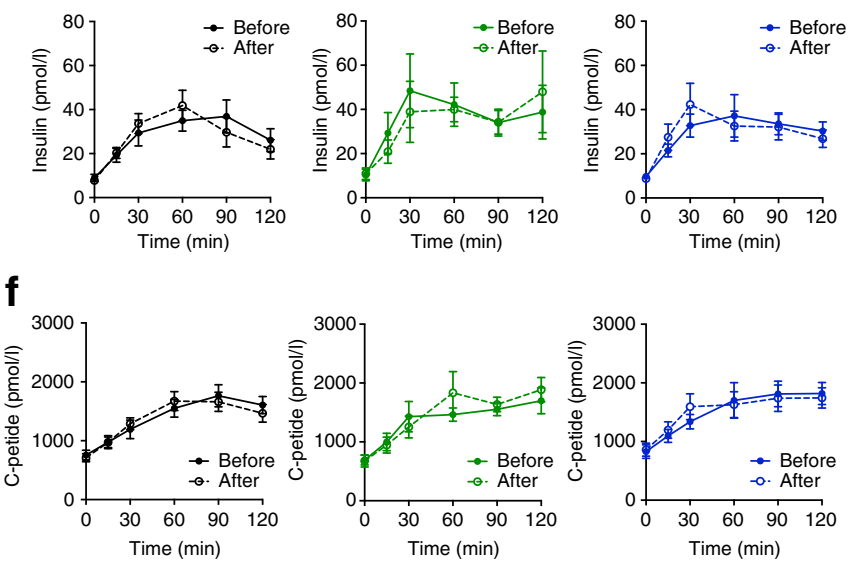

\section{h}
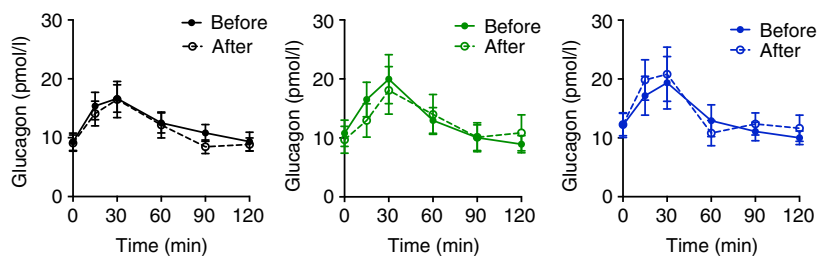

glucagon $(\mathbf{g}, \mathbf{h})$. (i-l) Measurements of insulinogenic index $(\mathbf{i}, \mathbf{j})$ and HOMA-IR $(\mathbf{k}, \mathbf{l})$ before and after a 12 week training intervention in participants with obesity (i, k) and participants with type 2 diabetes ( $\mathbf{j}$, l). Data represent mean values, error bars indicate SEM based on the full analysis set. Black data points, placebo; green data points, sitagliptin; blue

Following 12 weeks of exercise under IL-6 blockade, we did not observe an effect on meal-induced active GLP-1. Since the post-intervention tests were performed more than 4 weeks after the last infusion of tocilizumab it can be 
assumed that IL-6 was no longer antagonised [28]. Thus, the consequences of 12 weeks of IL- 6 blockade combined with exercise do not have a lasting influence on active GLP-1 or insulin.

The acute inhibitory effect of tocilizumab and the stimulatory effect of sitagliptin on active GLP-1 are likely to occur in response to every meal and every bout of exercise throughout the 12 week training intervention. It is therefore surprising that no clear effect on beta cell function (insulinogenic index) was observed. Yet, the proinsulin/insulin ratio, which is a good measure of beta cell health [29], improved with sitagliptin. Participants with type 2 diabetes also showed an improved proinsulin/insulin ratio following tocilizumab (not tested for statistical significance); this was in contrast to the findings in obese participants. In general, subgroup analysis seems to reveal a distinct effect of tocilizumab on a variety of variables related to postprandial blood glucose; however, further studies are needed as the present study was not sufficiently powered for subgroup analysis.

$\dot{V} O_{2 \max }$ improved both in obese and type 2 diabetic participants;, however, the improvements were rather limited. On average, participants trained 2.75 times per week and it is known that for some individuals four to five times per week is required to obtain cardiorespiratory improvements [30]. Training compliance was lower in the tocilizumab group compared with the placebo and sitagliptin groups. Blocking IL-6 signalling may reduce exercise capacity in humans, similar to what has been reported in whole body IL-6 knockout mice [31]. However, a recent study has shown that adaptations to an exercise training intervention in obese people on tocilizumab were similar to those in people on placebo [32].

In conclusion, this study demonstrates that IL-6 acutely regulates active GLP-1 in obese individuals and individuals with type 2 diabetes. Furthermore, IL-6 antagonism may have distinct effects in obese individuals compared with patients with type 2 diabetes.

\section{Limitations of the study}

The study population, more specifically, the subgroups, could be viewed as a limitation of the study. The subgroup numbers are rather low and limit the possibility of drawing strong conclusions. The absence of no-exercise control groups may be viewed as a limitation as it hinders conclusions in terms of distinguishing the effect of training from the effect of the drug. Along these lines, a prolonged washout period for sitagliptin might have been an advantage and allowed us to better separate the effect of sitagliptin alone from the effect of combining sitagliptin with exercise training. As metformin has been shown to increase GLP-1 levels [33], treatment of a subgroup of patients with metformin could be considered another limitation. However, it is unlikely that metformin affected the primary outcome, as treatment was initiated before study enrolment, evenly randomised and not changed over the study period.

Acknowledgements P. Zala (Department of Endocrinology, Diabetes and Metabolism, University Hospital Basel), S. Ruesch (Department of Endocrinology, Diabetes and Metabolism, University Hospital Basel), R. Rovsing (Centre for Physical Activity Research, Rigshospitalet) and H. Villumsen (Centre for Physical Activity Research, Rigshospitalet) are acknowledged for their technical assistance.

Data availability The datasets generated during and/or analysed during the current study are available from the corresponding author on reasonable request.

Funding The Centre for Physical Activity Research (CFAS) is supported by a grant from TrygFonden. During the study period, the Centre of Inflammation and Metabolism (CIM) was supported by a grant from the Danish National Research Foundation (DNRF55). HE was further supported by grants from the Danish Council for Independent Research (grant no. 12-132429) and the Novo Nordisk Foundation (project no. 959532028), and CIM/CFAS is a member of DD2 - the Danish Center for Strategic Research in Type 2 Diabetes (the Danish Council for Strategic Research, grant nos 09-067009 and 09-075724). This study was also supported by grants from the European Foundation for the Study of Diabetes and by the Swiss National Research Foundation.

Duality of interest The authors declare that there is no duality of interest associated with this manuscript.

Contribution statement HE and MYD conceived and designed the study. HE, ES, KT, LS, MPL, HH, AS-T, WOF, KK, NJWA, BKP and MBS acquired the data. MC analysed the data. HE, ES, MYD interpreted the data. HE, ES and MYD drafted the article. AS-T, WOF, BKP, MBS and NJWA provided resources. All co-authors critically revised the article. All co-authors approved the final version to be published. MYD is the guarantor of this work.

\section{References}

1. Pan XR, Li GW, Hu YH et al (1997) Effects of diet and exercise in preventing NIDDM in people with impaired glucose tolerance. The Da Qing IGT and Diabetes Study. Diabetes Care 20(4):537-544. https://doi.org/10.2337/diacare.20.4.537

2. Boule NG, Haddad E, Kenny GP, Wells GA, Sigal RJ (2001) Effects of exercise on glycemic control and body mass in type 2 diabetes mellitus: a meta-analysis of controlled clinical trials. JAMA 286(10):1218-1227. https://doi.org/10.1001/jama.286.10. 1218

3. Johansen MY, MacDonald CS, Hansen KB et al (2017) Effect of an intensive lifestyle intervention on glycemic control in patients with type 2 diabetes: a randomized clinical trial. JAMA 318(7):637-646. https://doi.org/10.1001/jama.2017.10169

4. Mauer J, Denson JL, Bruning JC (2015) Versatile functions for IL-6 in metabolism and cancer. Trends Immunol 36(2):92-101. https:// doi.org/10.1016/j.it.2014.12.008

5. Ostrowski K, Rohde T, Zacho M, Asp S, Pedersen BK (1998) Evidence that interleukin-6 is produced in human skeletal muscle during prolonged running. J Physiol 508(Pt 3):949-953. https://doi. org/10.1111/j.1469-7793.1998.949bp.x 
6. Pedersen BK, Febbraio MA (2008) Muscle as an endocrine organ: focus on muscle-derived interleukin-6. Physiol Rev 88(4):13791406. https://doi.org/10.1152/physrev.90100.2007

7. Martins C, Morgan LM, Bloom SR, Robertson MD (2007) Effects of exercise on gut peptides, energy intake and appetite. J Endocrinol 193(2):251-258. https://doi.org/10.1677/JOE-06-0030

8. Drucker DJ (2006) The biology of incretin hormones. Cell Metab 3(3):153-165. https://doi.org/10.1016/j.cmet.2006.01.004

9. Kreymann B, Williams G, Ghatei MA, Bloom SR (1987) Glucagon-like peptide-1 7-36: a physiological incretin in man. Lancet 2(8571):1300-1304. https://doi.org/10.1016/s01406736(87)91194-9

10. Nauck MA, Heimesaat MM, Behle K et al (2002) Effects of glucagon-like peptide 1 on counterregulatory hormone responses, cognitive functions, and insulin secretion during hyperinsulinemic, stepped hypoglycemic clamp experiments in healthy volunteers. J Clin Endocrinol Metab 87(3):1239-1246. https://doi.org/10.1210/ jcem.87.3.8355

11. Martins C, Kulseng B, King NA, Holst JJ, Blundell JE (2010) The effects of exercise-induced weight loss on appetite-related peptides and motivation to eat. J Clin Endocrinol Metab 95(4):1609-1616. https://doi.org/10.1210/jc.2009-2082

12. Chanoine JP, Mackelvie KJ, Barr SI, Wong AC, Meneilly GS, Elahi DH (2008) GLP-1 and appetite responses to a meal in lean and overweight adolescents following exercise. Obesity (Silver Spring) 16(1):202-204. https://doi.org/10.1038/oby.2007.39

13. Ellingsgaard H, Hauselmann I, Schuler B et al (2011) Interleukin-6 enhances insulin secretion by increasing glucagon-like peptide-1 secretion from L cells and alpha cells. Nat Med 17(11):14811489. https://doi.org/10.1038/nm.2513

14. Lang Lehrskov L, Lyngbaek MP, Soederlund L et al (2018) Interleukin-6 delays gastric emptying in humans with direct effects on glycemic control. Cell Metab 27(6):1201-1211.e3. https://doi. org/10.1016/j.cmet.2018.04.008

15. Matthews DR, Hosker JP, Rudenski AS, Naylor BA, Treacher DF, Turner RC (1985) Homeostasis model assessment: insulin resistance and beta-cell function from fasting plasma glucose and insulin concentrations in man. Diabetologia 28(7):412-419. https://doi.org/ 10.1007/bf00280883

16. Phillips DI, Clark PM, Hales CN, Osmond C (1994) Understanding oral glucose tolerance: comparison of glucose or insulin measurements during the oral glucose tolerance test with specific measurements of insulin resistance and insulin secretion. Diabet Med 11(3): 286-292. https://doi.org/10.1111/j.1464-5491.1994.tb00273.x

17. Wewer Albrechtsen NJ, Bak MJ, Hartmann B et al (2015) Stability of glucagon-like peptide 1 and glucagon in human plasma. Endocr Connect 4(1):50-57. https://doi.org/10.1530/EC-14-0126

18. Holm S (1979) A simple sequentially rejective multiple test procedure. Scand J Stat 6:65-70

19. Pinheiro JB, DebRoy S, Sarkar D, R Core Team (2017) Linear and nonlinear mixed effects models. R package version 3.1-131

20. Orskov C, Wettergren A, Holst JJ (1993) Biological effects and metabolic rates of glucagonlike peptide-1 7-36 amide and glucagonlike peptide-1 7-37 in healthy subjects are indistinguishable. Diabetes 42(5):658-661. https://doi.org/10.2337/diab.42.5. 658

21. Varin EM, Mulvihill EE, Beaudry JL et al (2019) Circulating levels of soluble dipeptidyl peptidase- 4 are dissociated from inflammation and induced by enzymatic DPP4 inhibition. Cell Metab 29(2):320334 e325. https://doi.org/10.1016/j.cmet.2018.10.001
22. Wewer Albrechtsen NJ, Mark PD, Terzic D et al (2019) Sacubitril/ valsartan augments postprandial plasma concentrations of active GLP-1 when combined with sitagliptin in men. J Clin Endocrinol Metab 104(9):3868-3876. https://doi.org/10.1210/jc.2019-00515

23. Faerch K, Torekov SS, Vistisen D et al (2015) GLP-1 Response to oral glucose is reduced in prediabetes, screen-detected type 2 diabetes, and obesity and influenced by sex: The ADDITION-PRO Study. Diabetes 64(7):2513-2525. https://doi.org/10.2337/db141751

24. Drucker DJ (2007) Dipeptidyl peptidase-4 inhibition and the treatment of type 2 diabetes: preclinical biology and mechanisms of action. Diabetes Care 30(6):1335-1343. https://doi.org/10.2337/ dc07-0228

25. Marathe CS, Rayner CK, Bound M et al (2014) Small intestinal glucose exposure determines the magnitude of the incretin effect in health and type 2 diabetes. Diabetes 63(8):2668-2675. https://doi. org/10.2337/db13-1757

26. Herman GA, Stevens C, Van Dyck K et al (2005) Pharmacokinetics and pharmacodynamics of sitagliptin, an inhibitor of dipeptidyl peptidase IV, in healthy subjects: results from two randomized, double-blind, placebo-controlled studies with single oral doses. Clin Pharmacol Ther 78(6):675-688. https://doi.org/10.1016/j. clpt.2005.09.002

27. Bergman AJ, Stevens C, Zhou Y et al (2006) Pharmacokinetic and pharmacodynamic properties of multiple oral doses of sitagliptin, a dipeptidyl peptidase-IV inhibitor: a double-blind, randomized, placebo-controlled study in healthy male volunteers. Clin Ther 28(1):55-72. https://doi.org/10.1016/j.clinthera.2006.01.015

28. Ternant D, Bejan-Angoulvant T, Passot C, Mulleman D, Paintaud G (2015) Clinical pharmacokinetics and pharmacodynamics of monoclonal antibodies approved to treat rheumatoid arthritis. Clin Pharmacokinet 54(11):1107-1123. https://doi.org/10.1007/ s40262-015-0296-9

29. Ward WK, LaCava EC, Paquette TL, Beard JC, Wallum BJ, Porte D Jr (1987) Disproportionate elevation of immunoreactive proinsulin in type 2 (non-insulin-dependent) diabetes mellitus and in experimental insulin resistance. Diabetologia 30(9):698-702. https://doi. org/10.1007/bf00296991

30. Montero D, Lundby C (2017) Refuting the myth of non-response to exercise training: 'non-responders' do respond to higher dose of training. J Physiol 595(11):3377-3387. https://doi.org/10.1113/ JP273480

31. Faldt J, Wernstedt I, Fitzgerald SM, Wallenius K, Bergstrom G, Jansson JO (2004) Reduced exercise endurance in interleukin-6deficient mice. Endocrinology 145(6):2680-2686. https://doi.org/ 10.1210/en.2003-1319

32. Wedell-Neergaard AS, Lang Lehrskov L, Christensen RH et al (2018) Exercise-Induced changes in visceral adipose tissue mass are regulated by IL- 6 signaling: a randomized controlled trial. Cell Metab 29(4):844-855.e3. https://doi.org/10.1016/j.cmet. 2018.12.007

33. DeFronzo RA, Buse JB, Kim T et al (2016) Once-daily delayedrelease metformin lowers plasma glucose and enhances fasting and postprandial GLP-1 and PYY: results from two randomised trials. Diabetologia 59(8):1645-1654. https://doi.org/10.1007/s00125016-3992-6

Publisher's note Springer Nature remains neutral with regard to jurisdictional claims in published maps and institutional affiliations. 